\title{
Development of Measurement System for Quantitative Evaluation of Skillfulness of Lower Extremities
}

\author{
Kazunori Yamazaki * \\ Fujita Health University, \\ 1-98 Dengakugakubo Kutsukake-cho, Toyoake, Aichi, 470-1192, Japan \\ Dongjun Jin, Yoshifumi Morita, Noritaka Sato, Hiroyuki Ukai \\ Nagoya Institute of Technology, \\ Gokiso-cho, Showa-ku, Nagoya, Aichi, 466-8555, Japan \\ Kenji Kozakai, Satoru Shibata, Shigenori Onishi, Akihiro Ito, Daisuke Mizuno \\ Sanyo Machine Works, Ltd., \\ 1 Oka Okimura, Kitanagoya, Aichi, 481-8540, Japan \\ Tatsuya Hirai, Haruna Takeda, Yuka Sugiura \\ Nishio Hospital, \\ 22 Izumi-cho, Nishio, Aichi, 445-0824, Japan \\ ${ }^{*}$ Corresponding author.E-mail: ymzkk@fujita-hu.ac.jp \\ Tel: +81-562-93-9216
}

\begin{abstract}
The final goal of our research is to develop a measurement system for quantitative evaluation of the skillfulness of the lower extremities. In our previous work, we developed the measurement system of the foot movement on the floor with the sitting posture. In this study, we developed a quantitative evaluation method of the skillfulness of the lower extremities. We verified the reliability of the proposed quantitative evaluation method by calculating the intra-rater reliability for two trials $I C C(1,1)$ of the evaluation results.
\end{abstract}

Keywords: Rehabilitation system, Quantitative evaluation, Intra-rater reliability, Lower extremities, Skillfulness

\section{Introduction}

Sensorimotor integration function and proprioceptive control in the lower extremities are necessary for walking and skillful movement. The sensorimotor integration function and proprioceptive control of elderly and other types of patients can become impaired.
On the other hand, evaluation of therapeutic effects is only done subjectively by experienced doctors or therapists. Quantitative evaluation of therapeutic effects has not been established. Therefore, there is a strong need for quantitative evaluation of therapeutic effects in clinical rehabilitation sites. Moreover, an effective training method for improving walking ability and 
skillful movement is imperative.

Thus, the final goal of our research is to develop a measurement system and robot system for quantitative evaluation of the skillfulness of the lower extremities, which has long been desired in clinical rehabilitation sites. The skillfulness of the lower extremities is the ability to adjust the posture and motion of the body to achieve a desired action.

We think that the skillfulness can be evaluated using the accuracy and speed of the motion when a subject's foot is moved to follow a given target trajectory. In this study, we developed a measurement system comprising three target trajectory courses with different difficulty levels, to evaluate the skillfulness of the lower extremities.

The quantitative evaluation results should also have high reliability. This reliability can be divided into intrarater reliability and inter-rater reliability [1]. Intra-rater reliability is the degree of agreement among multiple repetitions of a diagnostic test performed by a single rater. Inter-rater reliability is the degree of agreement among raters. The intra-class correlation (ICC) has been widely used to evaluate the intra-rater reliability in rehabilitation medicine.

In this study, we developed a quantitative evaluation method to assess the skillfulness of the lower extremities. For this purpose, we used the measurement system we developed in our previous work and redesigned the target trajectories of foot movement. Moreover, we verified the reliability of the proposed quantitative evaluation method by calculating the intrarater reliability for two trials $\operatorname{ICC}(1,1)$.

\section{Measurement system for quantitative evaluation of skillfulness of lower extremities}

\subsection{Hardware and operation procedure}

We have developed the measurement system of the foot movement on the floor with the sitting posture, as shown in Fig. 1. The hardware consists of a laptop PC, training device, and training board [2]. A PC wireless mouse is installed in the training device. The mouse is used as a low-cost position sensor. The pointer corresponding to the position of the training device is displayed on the monitor. We designed the system so that the motion of the pointer on the monitor and the motion of the foot on the training board would be the same size.
In the training, the subject moves the pointer displayed on the monitor by moving the training device in the sitting posture. During this training, the subject watches the monitor of the laptop PC and does not watch their lower extremities directly. The subject can perform the training by playing a courses displayed on the laptop PC monitor. The therapist can evaluate the motion using the data acquired during the training.

There is little risk of the patient overturning, because this system is operated in the sitting posture. Moreover, there are no dangers associated with a runaway training device because the system does not have any actuators. Therefore, this system can be easily introduced in the early stages of rehabilitation because of the high safety of this system.

\subsection{Software}

We developed three courses as target trajectories: a straight course, circular course, and star-shaped course, as shown in Fig. 2. The parameters, such as the length of the courses were designed so that the difficulties of the three courses were different. A depiction of the software is given in Fig. 3.

The subject moves the pointer displayed on the monitor quickly and accurately along the target trajectory. The results of the assessment of the skillfulness of the lower extremities are expressed in terms of speed and accuracy. The speed $t[\mathrm{~s}]$ is given by the travelling time for each course. The accuracy $S$ [pixel] is the area that is surrounded by the pointer trajectory and the target trajectory.

The extension of the knee joint is primarily used to follow the target trajectory in the straight course. On the other hand, in the circular and star-shaped courses, the complex motion that is a mixture of the extension of the

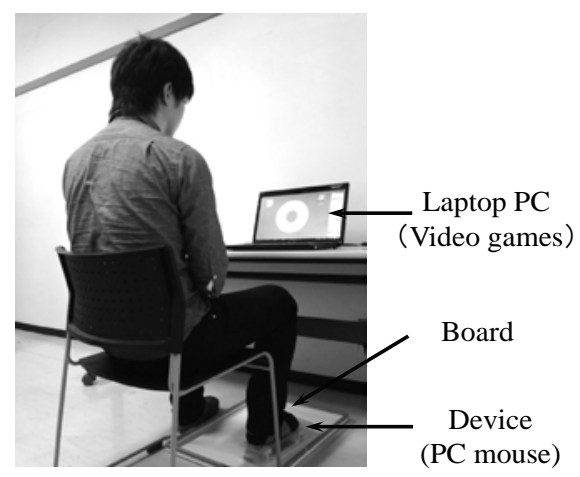

Fig. 1. Measurement system of the foot movement on the floor with the sitting posture. 
knee joint and the internal rotation of the hip joint is necessary. In addition, in the star-shaped course, more time is required for switching the direction of the motion than in the circular course. Therefore, each course is designed to have a different difficulty.

When the software starts, it will display a control panel on the monitor. In the control panel, the therapist inputs the subject's information (user name, ID, height, weight, Body Mass Index (BMI), dominant foot, remarks) and the location on HDD to save the data as a CSV file, at first. If this information is not input, measurement data is only saved to the desktop of the PC. At each sampling point, the $\mathrm{X} \& \mathrm{Y}$ coordinates of the pointer are measured and saved. The sampling frequency is $100 \mathrm{~Hz}$. After inputting the subject's information and the location to which on HDD, the therapist selects the course.

To check the results during/after the trials, the system shows the spent time from the start of each trial and the time that is counted up when the pointer is off the course, as the feedback to the subject. At this point, information can be eliminated from the control panel, if the therapist wants. In addition, the system can display the pointer trajectory overlaid on the target trajectory

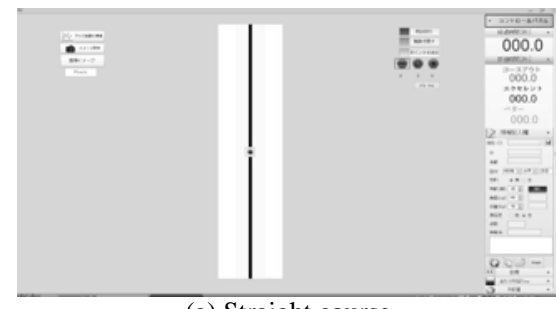

(a) Straight course

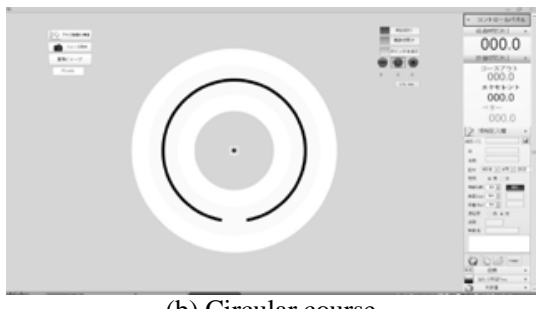

(b) Circular course

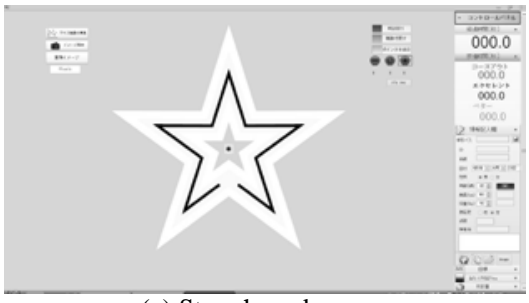

(c) Star-shaped course

Fig. 2. Three courses for evaluation. after the training, as shown in Fig. 4(a). The system can also display the area that is surrounded by the pointer trajectory and the target trajectory, as shown in Fig. 4(b).

\section{Verifying the intra-rater reliability}

\subsection{Method}

To verify the intra-rater reliability of the measurement system, we performed some experiments. The subjects were fourteen healthy young persons. The age was $22 \pm 2.4$ years old. The height was $166 \pm 8.3 \mathrm{~cm}$. The dominant foot of all subjects was right.

The subject was seated so that the distance between the subject's eyes and the PC monitor was $0.7 \mathrm{~m}$. The training device was attached to the subject's left foot. The height of the seat surface was adjusted so that the knee joint angle was 90 degree in the sitting position. The subjects performed tasks consisting of the three courses. The subjects were instructed to move the left foot along the black line (the desired trajectory) as

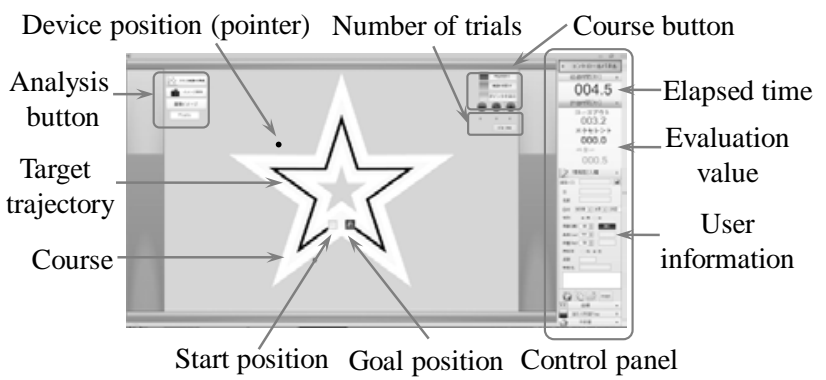

Fig. 3. Explanatory drawing of the user interface.

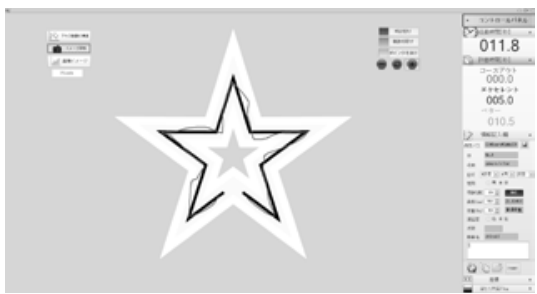

(a) Pointer trajectory and target trajectory

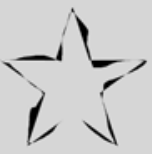

(b) Area surrounded by the pointer trajectory and the target trajectory

Fig. 4. Evaluation results. 
quickly and precisely as possible, not to move the body, and to move the left foot smoothly on the training board. The subjects performed the tasks two times for each course. After finishing each task, the result was not informed of the subject.

We used data from the three courses, with two repetitions for each person. The $\operatorname{ICC}(1,1)$ of the speed $t$ and the accuracy $S$ were calculated using the Eq. (1).

$$
\begin{aligned}
\operatorname{ICC}(1,1) & =\frac{B M S-W M S}{B M S+(k-1) W M S} \\
B M S & =\frac{\sum_{i=1}^{N} k\left(\bar{x}_{i}-\bar{x}\right)^{2}}{N-1}, \quad W M S=\frac{\sum_{i=1}^{N} \sum_{j=1}^{k}\left(x_{i j}-\bar{x}_{i}\right)^{2}}{N(k-1)}
\end{aligned}
$$

where $B M S$ is the variance between subjects, WMS is the variance in each subject, $k$ is the number of measurements, $N$ is the number of subjects, $\bar{x}$ is the mean value of the data from $N$ subjects and $k$ times, $\bar{x}_{i}$ is the mean value of the data from $k$ times of Subject $i$, and $x_{i j}$ is the data from Subject $i$ at time $j$. In this study, $k=2$ and $N=14$.

The criterion methods for $\operatorname{ICC}(1,1)$ have been reported [3][4][6]. In Ref. [3], the numbers were set such that 0.00-0.20: Slight, 0.21-0.40: Fair, 0.41-0.60: Moderate, 0.61-0.80: Substantial, and 0.81-1.00: Almost perfect. On the other hand, in Ref. [4], the numbers were reported such that more than 0.6: Possible, more than 0.7: Normal (OK), more than 0.8: Good, and more than 0.9 : Great.

Previous studies showed that if the $\operatorname{ICC}(1,1)$ is more than 0.7 , then the reliability is defined as good.

\subsection{Results and discussion}

The BMS and the WMS of the speed $t$ and accuracy $S$ for the three courses are listed in Table 1. From these values, the $\operatorname{ICC}(1,1) \mathrm{s}$ were calculated. The $\operatorname{ICC}(1,1) \mathrm{s}$ of the speed $t$ were $0.951,0.982$, and 0.984 for the straight course, the circular course, and the star-shaped course, respectively. The $\operatorname{ICC}(1,1)$ s of the accuracy $S$ were $0.265,0.924$, and 0.711 for the straight course, circular

Table 1. Results of BMS and WMS.

\begin{tabular}{|c|c|c|c|c|}
\hline \multirow{2}{*}{} & \multicolumn{3}{|c|}{ Three evaluating courses } \\
\cline { 2 - 5 } & $\begin{array}{c}\text { (a) Straight } \\
\text { course }\end{array}$ & $\begin{array}{c}\text { (b) Circular } \\
\text { course }\end{array}$ & $\begin{array}{c}\text { (c) Star-shaped } \\
\text { course }\end{array}$ \\
\hline \multirow{2}{*}{$\begin{array}{c}\text { speed } \\
t\end{array}$} & $B M S$ & $4.01 \times 10^{1}$ & $2.10 \times 10^{2}$ & $2.97 \times 10^{2}$ \\
\cline { 2 - 5 } & $W M S$ & $1.01 \times 10^{0}$ & $1.95 \times 10^{0}$ & $2.42 \times 10^{0}$ \\
\hline $\begin{array}{c}\text { accuracy } \\
S\end{array}$ & $B M S$ & $1.17 \times 10^{7}$ & $1.29 \times 10^{8}$ & $6.01 \times 10^{7}$ \\
\hline
\end{tabular}

course, and star-shaped course, respectively.

On the basis of these results, the reliabilities of the speed $t$ and the accuracy $S$ were confirmed for the circular and star-shaped courses. On the other hand, the reliability of the accuracy $S$ was not confirmed in the straight course. The reason for this can be determined from Table 1 and Eq. (1). In the case of the accuracy $S$ in the straight course, the ratio of BMS and WMS was small compared to the other cases. This means that the variance between the subjects was small and the variance in each subject was large. It is probable that the reason for this was that the straight course was easy for the healthy subjects.

As a result, it was confirmed that the developed measurement system has sufficient reliability in both the circular and star-shaped courses.

\section{Conclusion}

We developed a measurement system to assess the skillfulness of the lower extremities with a straight course, a circular course, and a star-shaped course. The quantitative evaluation method for the lower extremities in both the circular and star-shaped courses has sufficient reliability.

Future work will involve the verification of the reliability of the proposed quantitative evaluation method for elderly persons and subjects with reduced skillfulness of the lower extremities.

\section{References}

1. Shrout PE, Fleiss JL, Intraclass Correlations: Uses in Assessing Rater Reliability, Psychological Bulletin, 86 (1979) 420-428

2. Yamazaki K, Morita Y, Sato N, Ukai H, Nagaya M, Hayashi T, Ito T , Sakai Y, Quantitative Evaluation System of Sensorimotor Integration Function and Proprioception of Lower extremities -Verification Effectiveness for surgical patients with intramedullary spinal cord tumor-, The Japan Society for Welfare Engineering, 15(2) (2013) 44-49 (Article in Japanese)

3. Landis JR, Koch GG, The measurement of observer agreement for categorical data, Biometrics, 33 (1977) 159-174

4. Kuwabara Y, Saito T, Inagaki Y, Evaluation of intra- and inter-observer reliability, Kokyu To Junka, 41(10) (1993) 945-952 (Article in Japanese)

5. Portney LG, Watkins MP, Foundations If clinical research-Applications to practice, Appleton \& Lange, USA (1993) 505-516 